\title{
Nuclear multifragmentation within the framework of different statistical ensembles
}

\author{
C.E. Aguiar, R. Donangelo, and S.R. Souza \\ Instituto de Fisica, Universidade Federal do Rio de Janeiro \\ Cidade Universitária, CP 68528, 21941-972 Rio de Janeiro, Brazil
}

(Dated: January 2, 2018)

\begin{abstract}
The sensitivity of the Statistical Multifragmentation Model to the underlying statistical assumptions is investigated. We concentrate on its micro-canonical, canonical, and isobaric formulations. As far as average values are concerned, our results reveal that all the ensembles make very similar predictions, as long as the relevant macroscopic variables (such as temperature, excitation energy and breakup volume) are the same in all statistical ensembles. It also turns out that the multiplicity dependence of the breakup volume in the micro-canonical version of the model mimics a system at (approximately) constant pressure, at least in the plateau region of the caloric curve. However, in contrast to average values, our results suggest that the distributions of physical observables are quite sensitive to the statistical assumptions. This finding may help deciding which hypothesis corresponds to the best picture for the freeze-out stage.
\end{abstract}

PACS numbers: 25.70.Pq, 24.60.-k 


\section{INTRODUCTION}

The thermodynamics of the multifragment emission in nuclear reactions has been intensively investigated both experimentally $[1,[2,3,4$, 5] and theoretically [6, 7, 8, 9, 10, 11, 12, 13, 14, 15, 16, 17, 18, 19, 20, 21]. Particularly, the qualitative aspects of the nuclear caloric curve, such as the existence of a plateau, has been strongly debated 1,4, 15, 22, 23, 24, 25, 26, 27, 28, 29, 30, 31, 32, 33]. The controversy related to the determination of this quantity is mainly due to the great difficulties involved in the measurement of the excitation energy and the breakup temperature of the fragmenting system [25]. Therefore, the existence of a liquid-gas phase transition in nuclear matter could not be clearly established or ruled out yet. Furthermore, several statistical calculations, supported by experimental analyses [4], suggest that the heat capacity at constant pressure, $C_{p}$, can assume negative values under certain conditions [15, 16, 18, 20, 34, 35] whereas other considerations have led to opposite conclusions [10, 13].

Theoretical aspects related to the smallness of nuclear systems have also been discussed and could influence conclusions drawn from statistical calculations based upon different constraints 8]. More specifically, the equivalence of statistical ensembles is expected to be valid only for very large systems, i.e. in the thermodynamical limit. Of course, this condition is not fulfilled in the reactions we discuss here. In spite of this, statistical approaches are very successul in quantitatively describing many features of nuclear multifragmentation [20, 21].

In this work, we compare the predictions of the Statistical Multifragmentation Model (SMM) 34, 36, 37], concentrating on its micro-canonical, canonical and isobaric versions. By comparing them, one actually confronts two different pictures for the freeze-out stage. In the canonical and in the micro-canonical frameworks, the break-up volume is fixed, so that its properties are dictated only the by range of the nuclear forces. The underlying assumption in the isobaric ensemble is that the freeze-out stage is associated with a given collision rate and, therefore, the break-up volume is allowed to fluctuate from one microstate to another. Since the freeze-out configuration can be rather different in both cases, one expects to find characteristic fingerprints for some observables.

In sect. II] we give a detailed review of the different versions of the SMM used in this work and, in particular, of the isobaric ensemble. The comparison among their predictions is discussed in sect. III. We conclude in sect. IV with a brief summary and with suggestions 
for experimental analysis.

\section{THE MODEL}

The SMM is based on a scenario in which a hot and compressed source expands, undergoing a prompt statistical breakup at a density smaller than that of normal nuclear matter [36]. The different fragmentation modes $\{f\}$ are weighed according to their statis-

tical factors. The latter depend on the statistical ensemble adopted [21, 36, 38], although the essential ingredients of the model do not vary. We present below the three statistical versions used in this work.

\section{A. The Microcanonical Ensemble}

In the microcanonical version [34, 36, 37], each fragmentation mode $f$ of an excited source with mass and atomic numbers $A_{0}$ and $Z_{0}$, respectively, must strictly obey the following mass, charge and energy constraints:

$$
\begin{aligned}
& A_{0}=\sum_{\{A Z\}} N_{A Z}^{f} A, \\
& Z_{0}=\sum_{\{A Z\}} N_{A Z}^{f} Z,
\end{aligned}
$$

and

$$
-B_{A_{0}, Z_{0}}+E^{*}=C_{C} \frac{Z_{0}^{2}}{A_{0}^{1 / 3}}\left(\frac{V_{0}}{V}\right)^{1 / 3}+\sum_{\{A, Z\}} N_{A, Z}^{f} E_{A, Z}
$$

where $N_{A, Z}^{f}$ represents, within a fragmentation mode $f$, the multiplicity of a fragment with mass and atomic numbers $A$ and $Z$, respectively, $B_{A_{0}, Z_{0}}$ stands for the binding energy of the source, $E^{*}$ is the excitation energy deposited into the system and $T$ its breakup temperature. The first term on the right hand side of the equation above corresponds to the Coulomb energy of a homogeneously charged sphere at breakup volume $V$, whereas $V_{0}$ is the source's volume at normal nuclear density. The fragment energy $E_{A, Z}(T, V)$ :

$$
E_{A, Z}(T, V)=-B_{A, Z}+E_{A, Z}^{K}(T)+E_{A, Z}^{*}(T)+E_{A, Z}^{C}(V)
$$


has contributions from its binding energy $B_{A, Z}$, translational motion $E_{A, Z}^{K}(T)$, internal excitation energy $E_{A, Z}^{*}(T)$, and from the remaining Coulomb terms, $E_{A, Z}^{C}(V)$, which contribute to the total Coulomb energy of the system in the Wigner-Seitz approximation [36, 39]. Throughout this paper, we use the Liquid Drop formula adopted in ref. [37]:

$$
\begin{aligned}
B_{A, Z} & =w_{0} A-\beta_{0} A^{2 / 3}-C_{C} \frac{Z^{2}}{A^{1 / 3}} \\
& -K_{\text {asym }} \frac{(A-2 Z)^{2}}{A} /\left[1+\frac{9}{4} \frac{K_{\text {asym }}}{Q_{\text {asym }} A^{1 / 3}}\right]
\end{aligned}
$$

for all fragments with $A>4$. Empirical binding energies are used for lighter nuclei. The parameters entering in the expression above are $w_{0}=16.0 \mathrm{MeV}, \beta_{0}=18.0 \mathrm{MeV}, C_{C}=$ $0.737 \mathrm{MeV}, K_{\text {asym }}=30.0 \mathrm{MeV}$, and $Q_{\text {asym }}=35.0 \mathrm{MeV}$. The remaining energy terms in Eq. (41) read:

$$
\begin{gathered}
E_{A, Z}^{K}(T)=\frac{3}{2} T, \\
E_{A, Z}^{*}(T)=\frac{T^{2}}{\epsilon_{0}} A+\left(\beta(T)-T \frac{d \beta}{d T}-\beta_{0}\right) A^{2 / 3}, \\
\beta(T)=\left[\frac{T_{c}^{2}-T^{2}}{T_{c}^{2}+T^{2}}\right]^{5 / 4},
\end{gathered}
$$

and

$$
E_{A, Z}^{C}(V)=-C_{C} \frac{Z^{2}}{A^{1 / 3}}\left(\frac{V_{0}}{V}\right)^{1 / 3},
$$

where $\epsilon_{0}=16.0 \mathrm{MeV}$ and $T_{c}=18.0 \mathrm{MeV}$ is the critical temperature above which the surface tension vanishes. Light nuclei, $A<5$, are treated as particles without internal degrees of freedom. Therefore, they only contribute to the total energy through the binding and kinetic energies, except for alpha particles in which case one retains the bulk term in Eq. (77) to account for its particle-stable excited states.

The energy conservation stated by Eq. (31) allows one to determine the microcanonical temperature $T_{f}$ for each fragmentation mode $f$. Therefore, $T_{f}$ fluctuates from one partition 
to the other. The statistical weight $w_{f}$ of a fragmentation mode is given by its corresponding number of states:

$$
w_{f}=\exp \left(S_{f}\right)
$$

where $S_{f}$ stands for the entropy, which is obtained from the sum of the contributions due to each fragment. It is related to the total energy $E$ and the Helmholtz free energy $F$ through the standard thermodynamical expression:

$$
F=E-T S
$$

and

$$
S(T, V)=-\frac{\partial F}{\partial T}(T, V) .
$$

The Helmholtz free energy for a fragmentation mode $f$ can be written as:

$$
\begin{aligned}
F_{f}(T, V) & =F_{f}^{*}(T)+F_{f}^{\text {trans }}(T, V)+F_{f}^{C}(V) \\
& -\sum_{\{A, Z\}} N_{A, Z}^{f} B_{A, Z}
\end{aligned}
$$

where

$$
\begin{gathered}
F_{f}^{*}(T)=\sum_{\{A, Z\}} N_{A, Z}^{f} F_{A, Z}^{*}(T) \\
=\sum_{\{A, Z\}} N_{A, Z}^{f}\left[-\frac{T^{2}}{\epsilon_{0}} A+\left[\beta(T)-\beta_{0}\right] A^{2 / 3}\right] \\
F_{f}^{\text {trans }}(T, V)=T \sum_{\{A, Z\}}\left[-N_{A, Z}^{f} \log \left(\frac{g_{A, Z} V_{\text {free }}}{\lambda_{T}^{3}}\right)\right. \\
\left.+\log \left(N_{A, Z}^{f} !\right)\right] \\
F_{C}^{f}(V)=a_{C}^{f}\left(\frac{V_{0}}{V}\right)^{1 / 3},
\end{gathered}
$$


and

$$
a_{C}^{f}=C_{C}\left[\frac{Z_{0}^{2}}{A_{0}^{1 / 3}}-\sum_{\{A, Z\}} N_{A, Z}^{f} \frac{Z^{2}}{A^{1 / 3}}\right] .
$$

In the above equations, $g_{A, Z}$ stands for the the spin degeneracy factor of the fragment. It is assumed to be equal to unity for fragments which have internal degrees of freedom whereas empirical ground state values are used for the others. The free volume is related to $V$ by:

$$
V_{\text {free }}=V-V_{0}=V_{0} \chi, \quad 0 \leq \chi<\infty
$$

and $\lambda_{T}=\sqrt{2 \pi \hbar^{2} / m_{n} A T}$, where $m_{n}$ corresponds to the mass of the nucleon.

The mean value of any physical observable $\langle O\rangle$ is obtained by weighing its value in each partition by the corresponding number of states $w_{f}$ :

$$
\langle O\rangle_{\text {Micro }}=\frac{\sum_{f} w_{f} O_{f}}{\sum_{f} w_{f}} .
$$

In particular, the average pressure $\langle P\rangle$ can be calculated through:

$$
P_{f}=-\frac{\partial F_{f}}{\partial V}=\frac{\left(M_{f}-1\right) T_{f}}{V_{\text {free }}}+\frac{a_{C}^{f}}{3} \frac{V_{0}^{1 / 3}}{V^{4 / 3}} .
$$

The kinetic contribution is proportional to $M_{f}-1$, rather than to the total multiplicity, $M_{f}=\sum_{\{A, Z\}} N_{A, Z}$, as the center of mass is kept at rest. Even if it is not explicitly stated in the formulae, this condition is consistently imposed in all the versions of the model. Therefore, Eq. (3), for instance, is actually modified to take it into account.

Since, for large nuclei, the number of different fragmentation modes is huge [37], we make a Monte Carlo sample of the most important partitions. As the sampling method does not select partitions with equal probability, the statistical weight $w_{f}$ is corrected for this bias 37]. This procedure is also adopted in the canonical and in the isobaric ensembles presented below.

Finally, a multiplicity dependent free volume has been introduced in refs. [34, 37]:

$$
\chi_{f}=\left[1+\frac{D}{2 r_{0} A_{0}^{1 / 3}}\left(M_{f}^{1 / 3}-1\right)\right]^{3}-1,
$$

where $D=2.3 r_{0}$. This causes the breakup volume to fluctuate from one partition to the other. 


\section{B. The Canonical Ensemble}

If the breakup temperature of the system $T$ is fixed, instead of the excitation energy of the source, besides its breakup volume, mass and atomic numbers, the canonical ensemble is the best suited statistical treatment. The partition function associated with a given fragmentation mode reads:

$$
Z_{f}^{C}(T, V)=\exp \left[\frac{-F_{f}(T, V)}{T}\right] .
$$

Then, the average value of a physical observable is calculated through:

$$
\langle O\rangle_{\mathrm{C}}=\frac{\sum_{f} Z_{f}^{C} O_{f}}{\sum_{f} Z_{f}^{C}} .
$$

Since the same essential ingredients are used in all the statistical ensembles, the Helmholtz free energy $F$ is also given by Eq. (13). Therefore, Eq. (20) still holds for the pressure in a given fragmentation mode. The total energy of the system can be calculated though Eqs. (11) and (12), so that it is given by:

$$
\begin{aligned}
E_{f} & =\frac{3}{2} T\left(M_{f}-1\right)+\sum_{\{A Z\}} N_{A Z}\left[-B_{A Z}+E_{A Z}^{*}(T)\right] \\
& +C_{C}^{f}\left(\frac{V_{0}}{V}\right)^{1 / 3}
\end{aligned}
$$

which now fluctuates from one fragmentation mode to the other since the temperature is fixed. The excitation energy is then given by:

$$
E_{f}^{*}=E_{f}+B_{A_{0} Z_{0}} .
$$

\section{The Isobaric Ensemble}

If one now fixes the pressure $P$, instead of the breakup volume, the canonical ensemble can be modified to keep the pressure constant for all microstates. In this case, the partition function becomes:

$$
Z_{f}^{\text {Iso }}(T, P)=\int_{V_{0}}^{\infty} d V Z_{f}^{C}(T, V) \exp \left(-\frac{P V}{T}\right)
$$


which, by using Eqs. (13] [16), can be rewritten as:

$$
Z_{f}^{\mathrm{Iso}}(T, P)=\tilde{Z}_{f}(T, P) I_{f}(T, P) .
$$

In the equation above, we have defined:

$$
\begin{gathered}
I_{f}(T, P)=\int_{0}^{\infty} d \chi \chi^{M_{f}-1} \exp \left(-\frac{Q_{f}(\chi, P)}{T}\right), \\
Q_{f}(\chi, P)=P V_{0} \chi+\frac{a_{C}^{f}}{(1+\chi)^{1 / 3}}, \\
\tilde{Z}_{f}(T, P)=\frac{V_{0}^{M_{f}}}{\lambda_{T}^{3\left(M_{f}-1\right)}} \exp \left(-\frac{P V_{0}}{T}\right) \prod_{\{A, Z\}} \Gamma_{A, Z},
\end{gathered}
$$

and

$$
\Gamma_{A, Z}=\frac{\left(g_{A, Z} A^{3 / 2}\right)^{N_{A, Z}}}{N_{A, Z} !} \exp \left[\frac{N_{A, Z}}{T}\left[B_{A, Z}-F_{A, Z}^{*}(T)\right]\right] .
$$

Similarly to the former case, average values are obtained through:

$$
\langle O\rangle_{\text {iso }}=\frac{\sum_{f} Z_{f}^{\text {Iso }} O_{f}}{\sum_{f} Z_{f}^{\text {Iso }}} .
$$

The appropriate thermodynamical function for the isobaric ensemble is the Gibbs free energy $G$ :

$$
G=F+P V
$$

which is related to the partition function by:

$$
G_{f}(T, P)=-T \log \left[Z_{f}^{\mathrm{Iso}}(T, P)\right] .
$$

From it, one may obtain the average volume of the system, for a given fragmentation mode:

$$
\bar{V}^{f}=\frac{\partial G_{f}}{\partial P}=V_{0} \frac{I_{f}^{V}(T, P)}{I_{f}(T, P)},
$$

where

$$
I_{f}^{V}(T, P)=\int_{0}^{\infty} d \chi(1+\chi) \chi^{M_{f}-1} \exp \left(-\frac{Q_{f}(\chi, P)}{T}\right)
$$


The energy of the system corresponding to the breakup channel $f$ can then be written as:

$$
\begin{aligned}
E_{f} & =\frac{\partial}{\partial(1 / T)}\left(\frac{G_{f}}{T}\right)-P \bar{V}^{f} \\
& =P V_{0}\left[1+\frac{I_{f}^{E}(P, T)}{I_{f}(P, T)}-\frac{I_{f}^{V}(P, T)}{I_{f}(P, T)}\right]+\frac{3}{2}\left(M_{f}-1\right) \\
& +\sum_{\{A, Z\}} N_{A, Z}\left[-B_{A, Z}+E_{A, Z}^{*}(T)\right],
\end{aligned}
$$

so that

$$
E_{f}^{*}=E_{f}+B_{A_{0} Z_{0}},
$$

where

$$
\begin{aligned}
I_{f}^{E}(T, P)=\int_{0}^{\infty} & {\left[\chi+\frac{a_{C}^{f}}{P V_{0}} \frac{1}{(1+\chi)^{1 / 3}}\right] \chi^{M_{f}-1} } \\
& \exp \left(-\frac{Q_{f}(\chi, P)}{T}\right) d \chi .
\end{aligned}
$$

Owing to the Coulomb factor $(1+\chi)^{-1 / 3}$, all the integrals above have to be evaluated numerically for each fragmentation mode. Since the integrands are bell shaped, the gaussian quadrature method is very efficient.

Finally, from the above expressions, the entropy can be evalutated from the relation:

$$
S_{f}=\frac{E_{f}+P \bar{V}^{f}-G_{f}}{T} .
$$

\section{COMPARISON AMONG THE ENSEMBLES}

All the example calculations in this work are carried out for a $A=168$ and $Z=75$ system. The same breakup temperature is used in the isobaric and in the canonical calculations. We fixed the pressure, in the isobaric ensemble, at $p=0.114 \mathrm{MeV} / \mathrm{fm}^{3}$. Although our conclusions are not qualitatively affected by this particular choice, the results below show that this is a reasonable value and lies within the expected range for this system [40]. Since the micro-canonical ensemble requires the energy as input, we use the average excitation 
energy obtained in the isobaric ensemble, calculated through Eqs. (37, 38) and (32), for a given temperature. The breakup volume, which is a free parameter for the canonical and micro-canonical ensembles, is chosen in different ways, as explained below.

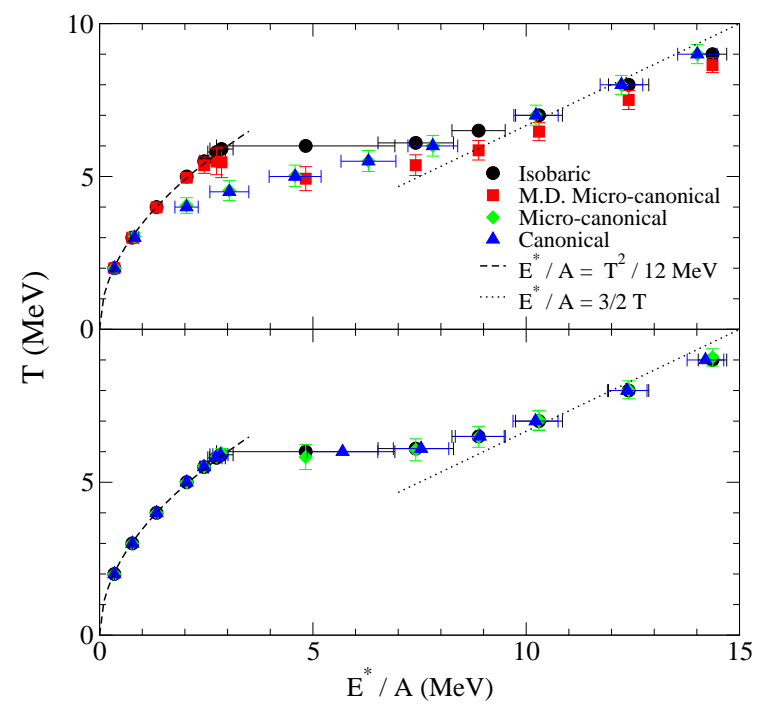

FIG. 1: Caloric curve predicted by the different ensembles. Upper panel: the breakup volume is kept constant for all energies for the canonical and the standard micro-canonical ensembles. Lower panel: the average breakup volume predicted by the isobaric calculation is used as input for the other ensembles. For details, see text.

Before we present the model results for the different ensembles, it is important to stress that the error bars in all the figures correspond to the standard deviations of the distributions, rather than to statistical fluctuations. Indeed, all the curves show a smooth behavior, even when the error bars are appreciably large. We included this information in all the pictures so as to provide more information about the distributions instead of only mean values.

We start by analysing the caloric curve predicted by the isobaric ensemble, which is depicted by the circles in Fig. 1, It shows that, at low temperatures $(T<6 \mathrm{MeV})$, the caloric curve follows the standard Fermi gas expression (represented by the dashed line in this picture), in agreement with previous calculations [34]. However, a sudden change takes place at $T=6 \mathrm{MeV}$ and one observes a fairly wide plateau, which signals a liquid-gas phase transition. For higher temperatures, the system tends to behave as a free gas, as also observed in ref. [34]. We have checked that the critical temperature depends on the 
pressure, but the essential features of the process remain the same.

It is well known that SMM predicts the existence of a plateau in the caloric curve only if the breakup volume, for a fixed excitation energy, is allowed to vary from one fragmentation mode to the other [34, 40, 41, 42]. Indeed, we also show, in the upper panel of Fig. 11 the results obtained using two versions of SMM. The one for which the breakup volume is determined, for each fragmentation mode, by Eqs. (18) and (21), is labelled "M.D. micro-canonical" (Multiplicity Dependent), to distinguish it from the "standard" micro-canonical version (with no additional labels) in which the breakup volume is fixed for all partitions at a given excitation energy. The results clearly show that the temperature increases monotonously if the breakup volume is kept fixed for all energies (diamonds) although a behavior similar to that predicted by the isobaric ensemble is observed in the other case (squares). In all calculations in which the breakup volume is the same for all energies, we adopt $V / V_{0}=3$, for both micro-canonical and canonical ensembles.

The predictions made by the canonical version of SMM are represented by the triangles in the upper panel of Fig. 1. An excelent agreement between the canonical and the microcanonical ensembles is obtained, showing the consistency of the model, as long as the relevent macroscopic quantites, such as breakup volume, average temperature/excitation energy, and pressure, are similar.

This expectation is reinforced through the comparison among the caloric curves predicted by the isobaric, standard micro-canonical, and canonical ensembles shown in the lower panel of Fig. 1. In this case, the breakup volume for the latter two ensembles, for each temperature/excitation energy, is taken to be the average value obtained in the isobaric calculation. It is clear that all the curves collapse into a single one. This implies that the most appropriate statistical scenario for nuclear multifragmentation may not be singled out by examining average values only.

To further clarify the aspects discussed above, we now investigate the average pressure predicted by the different versions of the model. The results shown in Fig. 2 are calculated from Eqs. (20)-(19) and Eqs. (20)-(23), for the micro-canonical and canonical ensembles, respectively. A steady increase of the pressure as a function of the excitation energy is observed, in the upper panel of this figure, for the standard micro-canonical and the canonical calculations. Since the breakup volume is the same for all excitation energies, this behavior is quite reasonable on physical grounds. On the other hand, fluctuations of the breakup 


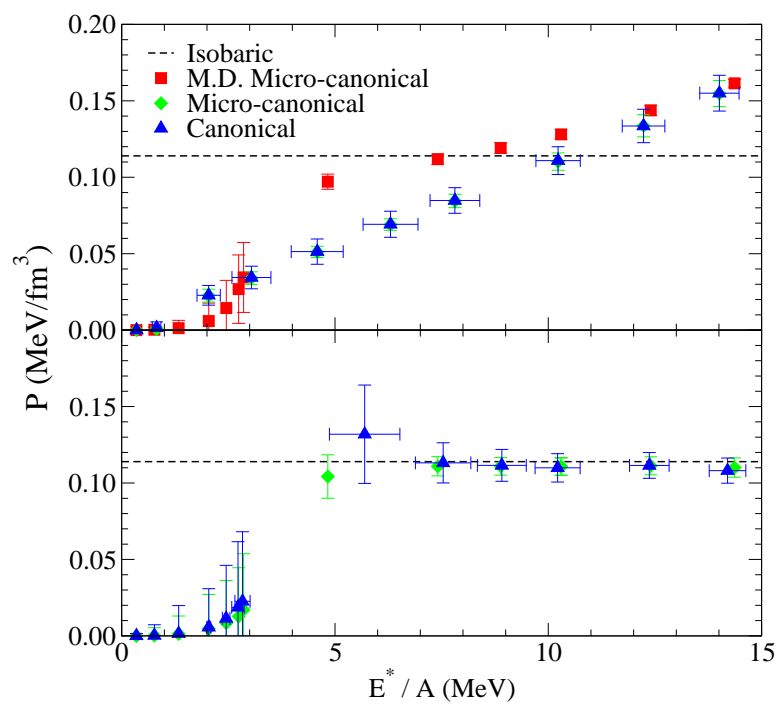

FIG. 2: Average pressure predicted by the different ensembles as a function of the excitation energy. In the upper panel the breakup volume is kept constant for all energies, except for the M.D. microcanonical version. The results displayed in the lower panel are obtained by using the average breakup volume predicted by the isobaric ensemble as input to the canonical and micro-canonical calculations.

volume, from one fragmentation mode to the other, introduced by Eqs. (18) and (21), lead to a weak energy dependence in the plateau region, as revealed by the results obtained employing the corresponding micro-canonical model, represented by the squares in the upper panel of this picture.

The role played by the volume change is emphasized by the results shown in the lower panel of the same picture. In this case, the breakup volume for the canonical and microcanonical calculations is chosen so that it corresponds to the average value obtained in the isobaric ensemble for each temperature. The pressure now stays fairly constant for $E^{*} / A>5 \mathrm{MeV}$. For lower excitation energies, it decreases owing to the influence of the large remnant, which, in this energy range, is almost always present.

The prediction of a plateau in the caloric curve and the similarity of the average pressure, in this region, found in the isobaric and the M.D. micro-canonical calculations, seem to indicate that the ad hoc multiplicity dependence, in the latter, Eqs. (18) and (21), leads to similar breakup volumes in both statistical ensembles. This is illustrated in Fig. 3, which displays the average breakup volume obtained with these two versions of the model. As expected, the predictions are very similar, although the micro-canonical ensemble gives 
smaller average breakup volumes at high energies. This explains why the average pressure increases for $E^{*} / A>10 \mathrm{MeV}$. A striking feature of the isobaric ensemble is the linear energy dependence of the breakup volume after the onset of multifragment emission. This is expected to happen for a gas with no internal degrees of freedom but, particularly at the plateau, the contribution from complex fragments is not negligible.

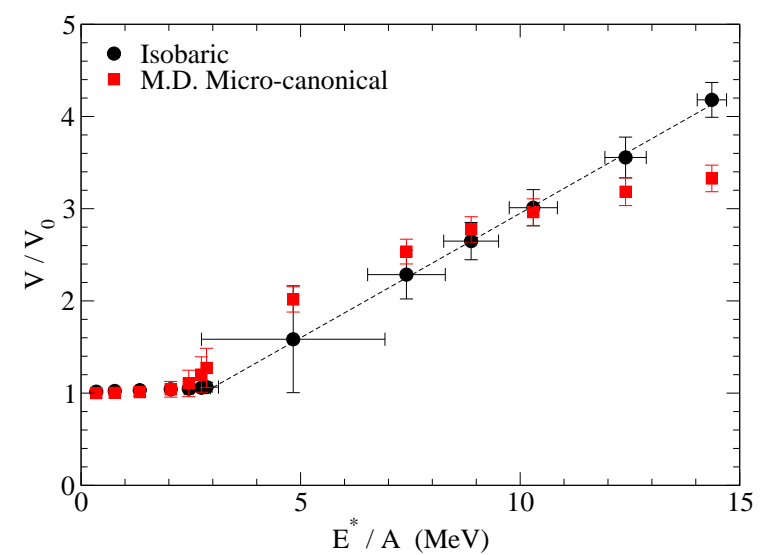

FIG. 3: Average breakup volume as a function of the excitation energy predicted by the isobaric and the micro-canonical ensembles. The dashed line just emphasizes the fairly linear dependence of the breakup volume on the excitation energy in the isobaric ensemble. For details see text.

Although the compatibility among the different versions of the model can be understood, some qualitative aspects still depend strongly upon the version one uses. This is demonstrated by the plot of the entropy as a function of the temperature in Fig. 4. In the upper panel, the canonical and the standard microcanonical calculations were carried out at fixed breakup volume for all temperatures. One sees that the entropy rises smoothly as the excitation energy increases. However, a backbending is observed in the results obtained with the M.D. microcanonical version. This led the authors of ref. [34] to predict negative heat capacities in the plateau region. However, our results (Fig. 2) show that the pressure is only approximately constant in the microcanonical calculations. Thus, one should be careful when drawing conclusions about $C_{p}$ from the entropy plot within this microcanonical calculation, since the microstates are not subject to a constant pressure.

On the other hand, from the results obtained with the isobaric ensemble, depicted by the circles in this picture, one can certainly assert that $C_{p}$ is very large, but always positive, in the plateau region. Nevertheless, the consistency of the model is not affected by this 


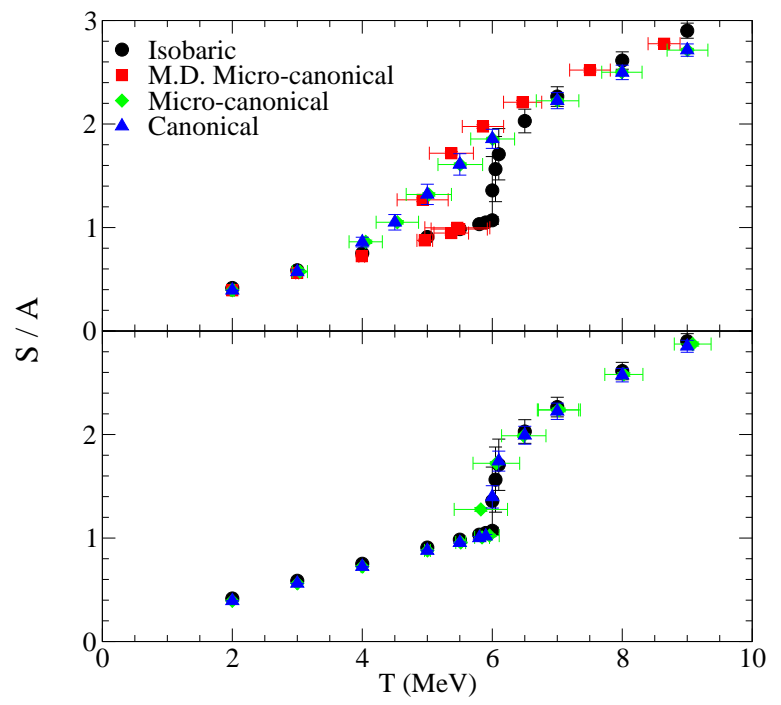

FIG. 4: Entropy per nucleon as a function of the temperature. Results corresponding to the canonical and the standard microcanonical ensembles are obtained at a constant volume for all temperatures in the upper panel, whereas the average values given by the isobaric ensemble were used as input in the lower panel. For details see text.

disagreement as is shown in the lower panel of Fig. 4, in which the breakup volume for the canonical and the microcanonical calculations are taken from the average values predicted by the isobaric ensemble at each temperature. Also in this case, a very good agreement among the ensembles is found. The microcanonical calculations still seem to predict negative $C_{p}$ in a narrow temperature region, although one should keep in mind the remarks above. The relevant point here is that all ensembles make very similar predictions under equivalent conditions.

Even though it is difficult to determine experimentally the heat capacity, other observables are very sensitive to the statistical assumptions and could, therefore, help to decide which picture is more convenient to describe the multiframent emission mechanism.

For instance, in Fig. 15, we show the isotopic distributions for a few selected cases obtained using the different versions of the model. One notices that the isobaric, canonical, and the standard microcanonical ensembles give very similar isotopic distributions. As above, the breakup volume for the canonical and the microcanonical models is taken from the isobaric calculations. In contrast, the isotopic distributions predicted by the M.D. microcanonical are systematically narrower. Since precise data are avaliable for many reactions [43], important 


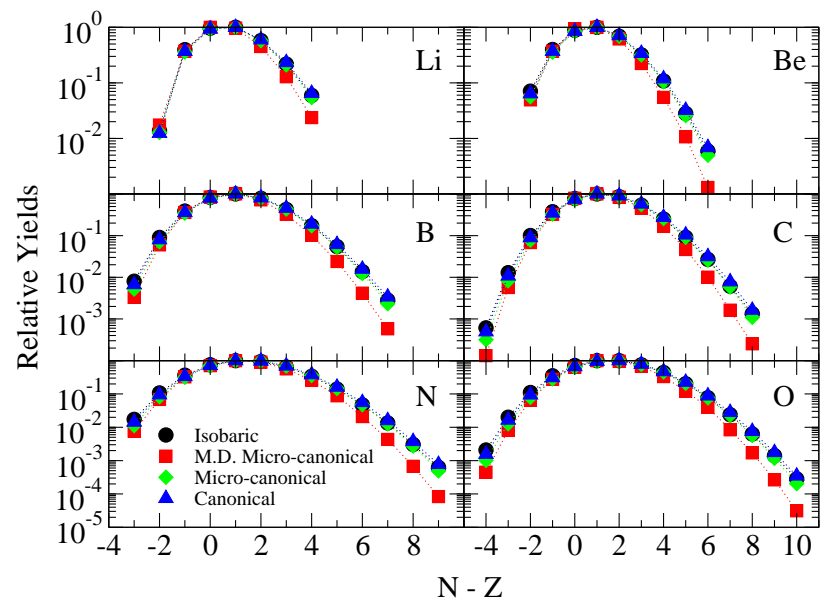

FIG. 5: Isotopic distributions predicted by the different ensembles. The yields are normalized relative to the maximum for each $Z$ value.

conclusions may be drawn from the study of this observable.

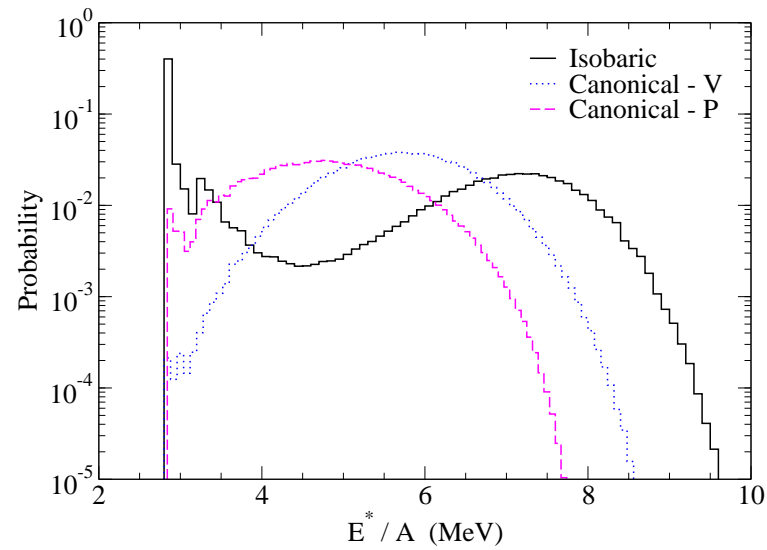

FIG. 6: Energy distribution obtained with the isobaric and canonical ensembles calculated at $T=6 \mathrm{MeV}$. For details see text.

Another key observable is the excitation energy distribution, which is shown in Fig. 6 for the isobaric and canonical ensembles at $T=6 \mathrm{MeV}$. As can be noticed, it turns out to be quite sensitive to the statistical assumptions. The canonical calculations have been performed in two different ways. In one case, the breakup volume corresponds to the average value obtained in the isobaric calculation and is labelled canonical-V. In the second canonical calculation, the breakup volume is chosen so that it gives the average 
pressure $p=0.114 \mathrm{MeV} / \mathrm{fm}^{3}$, and labelled canonical-P. These two canonical calculations agree qualitatively, as both give bell shaped distributions, which essentially differ by the mean values. On the other hand, when all the microstates are at the same pressure, the energy distribution has a narrow peak at low excitation energy and a smeared bump at high excitation energies, with a large gap between them. The low energy part of the distribution correspond to the presence of a large remnant (the liquid phase) in the breakup channel, whereas essentially light fragments are found at high energies (the gas phase).

This is illustrated in Fig. 17, which shows the results obtained with the isobaric and canonical ensembles. The canonical calculations are done in the manner just described. One should notice that the tendency to produce a large remnant in the breakup channel seems to be a pressure efffect as the canonical calculation which mimics the isobaric ensemble appears to produce heavy fragments and even shows a small bump near $A_{0}$. This behavior is not seen in the other canonical calculation.

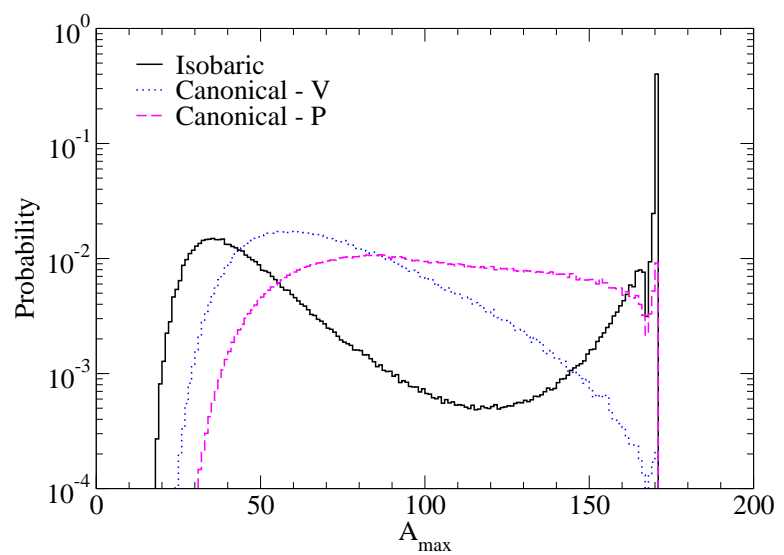

FIG. 7: Distribution of the largest fragment within each fragmentation mode obtained with the isobaric and canonical ensembles at $T=6 \mathrm{MeV}$.

\section{CONCLUDING REMARKS}

We have examined the sensitivity of the predictions of the SMM to its statistical assumptions. We found that the microcanonical, canonical, and isobaric implementations of SMM predict very similar caloric curves, and other (average) physical observables, provided 
macroscopic variables, such as temperature, excitation energy, and breakup volume, are the same in all versions of the model.

Although this is not the main point of this work, it is worth reporting that we found large values of $C_{p}$ at the plateau region of the caloric curve, but no indication of negative heat capacities in our isobaric calculations, in agreement with ref. 13]. We have concentrated on the $A_{0}=168, Z_{0}=75$ system, but we have also checked that this conclusion still holds for lighter systems, such as the ${ }^{108} \mathrm{Ag}$ nucleus, in contrast with other calculations [5, 15, 18, 34, 35]. More specifically, we observed negative $C_{p}$ only in the microcanonical calculations, as already reported in refs. [16, 20, 34]. Nevertheless, the predictions made by the isobaric ensemble should be more reliable since it is the only statistical ensemble in which all the microcospic states are subject to the same pressure, whereas, in the other cases, this condition if fulfilled only on the average. We should also mention that we did not investigate the sensitivity of this conclusion to the model parameters related to the binding and excitation energy of the source. As reported in ref. [18] these parameters may influence the mass region in which negative $C_{p}$ values are obtained in the statistical calculations.

We would like to stress the point that the distributions of physical observables, rather than their mean values, seem to be fairly sensitive to the statistical assumptions. The relevance of this conclusion goes beyond the fact that the equivalence of the statistical ensembles should hold only in the thermodynamical limit [8]. Indeed, some predictions made upon different assumptions agree very well, as long as the most relevant input global parameters do not differ appreciably. Strictly speeking, one may distinguish among the different calculations. However, we belive that experiments cannot be precise enough to single out one of them only by examining average values. Therefore, although nuclear systems are small and nuclear reactions cannot be fine tuned, the distributions of some physical observables should bear clear signatures related to the underlying statistical assumptions. Then, the analysis of such distributions may be valuable in establishing an appropriate scenario for the freeze-out stage in nuclear multifragmentation. 


\section{Acknowledgments}

We would like to acknowledge CNPq, FAPERJ, and the MCT/FINEP/CNPq (PRONEX) program, under contract \#41.96.0886.00, for partial financial support.

[1] Y. G. Ma, J. B. Natowitz, R. Wada, K. Hagel, J. Wang, T. Keutgen, Z. Majka, M. Murray, L. Qin, P. Smith, et al., Phys. Rev. C 71, 054606 (2005).

[2] M. Pichon, B. Tamain, R. Bougault, and O. Lopez, Nucl. Phys. A749, 93c (2005).

[3] J. B. Elliott, L. G. Moretto, L. Phair, G. J. Wozniak, S. Albergo, F. Bieser, F. P. Brady, Z. Caccia, D. A. Cebra, A. D. Chacon, et al., Phys. Rev. C 67, 024609 (2003).

[4] M.D'Agostino, R. Bougault, F. Gulminelli, M. Bruno, F. Cannata, Ph. Chomaz, F. Gramegna, I. Iori, N. Le Neindre, G. Margagliotti, et al., Nucl. Phys. A699, 795 (2002).

[5] R. P. Scharenberg, S. A. B. K.S̃rivastava, F. Bieser, F. P. Brady, Z. Caccia, D. A. Cebra, A. D. Chacon, J. L. Chance, Y. Choi, S. Costa, et al., Phys. Rev. C 64, 054602 (2001).

[6] C. B. Das, S. Das Gupta, W. G. Lynch, A. Z. Mekjian, and M. B. Tsang, Phys. Rep. 406, 1 (2005).

[7] J. B. Elliott, L. G. Moretto, and L. Phair, Phys. Rev. C 71, 024607 (2005).

[8] F. Gulminelli and Ph. Chomaz, Phys. Rev. C 71, 054607 (2005).

[9] S. K. Samaddar, J. N. De, and A. Bonasera, Phys. Rev. C. 71, 011601 (2005).

[10] X. Campi, K. Krivine, E. Plagnol, and N. Sator, Phys. Rev. C 71, R041601 (2005).

[11] A. Barrañón, J. Escamilla Roa, and J. A. López, Phys. Rev. C 69, 014601 (2004).

[12] C. M. Mader, A. Chappars, J. B. Elliott, L. G. Moretto, L. Phair, and G. J. Wozniak, Phys. Rev. C 68, 064601 (2003).

[13] L. G. Moretto, J. B. Elliott, L. Phair, and G. J. Wozniak, Phys. Rev. C 66, R041601 (2002).

[14] J. M. Carmona, J. Richert, and P. Wagner, Phys. Lett. B 531, 71 (2002).

[15] J. B. Elliott and A. S. Hirsch, Phys. Rev. C 61, 054605 (2000).

[16] Ph. Chomaz, V. Duflot, and F. Gulminelli, Phys. Rev. Lett. 85, 3587 (2000).

[17] C. B. Das, S. Das Gupta, and A. Z. Mekjian, Phys. Rev. C 68, 031601(R) (2003).

[18] C. B. Das, S. Das Gupta, and A. Z. Mekjian, Phys. Rev. C 68, 014607 (2003).

[19] C. B. Das and S. Das Gupta, Phys. Rev. C 64, 017601 (2001). 
[20] D. H. E. Gross, Phys. Rep. 279, 119 (1997).

[21] J. P. Bondorf, A. S. Botvina, A. S. Iljinov, I. N. Mishustin, and K. Sneppen, Phys. Rep. 257, 133 (1995).

[22] X. Campi, H. Krivine, and E. Plagnol, Phys. Lett. B 385, 1 (1996).

[23] J. Natowitz, R. Wada, K. Hagel, T. Keytgen, M. Murray, A. Makeev, L. Qin, P. Smith, and C. Hamilton, Phys. Rev. C 65, 034618 (2002).

[24] A. Ruangma, R. Laforest, E. Martin, E. Ramakrishnan, D. J. Rowland, M. Veselsky, E. M. Winchester, S. J. Yennello, L. Beaulieu, W. c. Hsi, et al., Phys. Rev. C 66, 044603 (2002).

[25] S. Das Gupta, A. Z. Mekjian, and M. B. Tsang, Adv. Nucl. Phys. 26, 91 (2001).

[26] J. Péter, Nuovo Cimento Soc. Ital. Fis. 111A, 977 (1998).

[27] K. Kwiatkowski, A. S. Botvina, D. S. Bracken, E. Foxford, W. A. Friedman, R. G. Korteling, K. B. Morley, E. C. Pollacco, V. E. Viola, and C. Volant, Phys. Lett. B 423, 21 (1998).

[28] V. Serfling, C. Schwarz, R. Bassini, M. Begemann -Blaich, S. Fritz, S. J. Gaff, C. Gross, G. Immé, I. Iori, U. Kleinevoss, et al., Phys. Rev. Lett. 80, 3928 (1998).

[29] H. F. Xi, G. J. Kunde, O. Bjarki, C. K. Gelbke, R. C. Lemmon, W. G. Lynch, D. Magestro, R. Popescu, R. Shomin, M. B. Tsang, et al., Phys. Rev. C 58, 2636 (1998).

[30] J. A. Hauger, S. Albergo, F. Bieser, F. P. Brady, Z. Caccia, D. A. Cebra, A. D. Chacon, J. L. Chance, Y. Choi, S. Costa, et al., Phys. Rev. Lett. 77, 235 (1996).

[31] Y. G. Ma, A. Siwer, J. Péter, F. Gulminelli, R. Dayras, L. Nalpas, B. Tamain, E. Vient, G. Auger, C. Bacri, et al., Phys. Lett. B 390, 41 (1997).

[32] L. G. Moretto, R. Ghetti, L. Phair, K. Tso, and J. G. Wozniak, Phys. Rev. Lett. 76, 2822 (1996).

[33] J. Pochodzalla, T. Möhlenkamp, T. Rubehn, A. Schüttauf, A. Wörner, E. Zude, M. Begermann-Blaich, T. Blaich, H. Emling, A. Ferrero, et al., Phys. Rev. Lett. 75, 1040 (1994).

[34] J. P. Bondorf, R. Donangelo, I. N. Mishustin, and H. Schulz, Nucl. Phys. A444, 460 (1985).

[35] S. K. Samaddar, J. N. De, and S. Shlomo, Phys. Rev. C 69, 064615 (2004).

[36] J. P. Bondorf, R. Donangelo, I. N. Mishustin, C. J. Pethick, H. Schulz, and K. Sneppen, Nucl. Phys. A443, 321 (1985).

[37] K. Sneppen, Nucl. Phys. A470, 213 (1987).

[38] A. S. Botvina, A. S. Iljinov, I. N. Mishustin, J. P. Bondorf, R. Donangelo, and K. Sneppen, 
Nucl. Phys. A475, 663 (1987).

[39] E. Wigner and F. Seitz, Phys. Rev. 46, 509 (1934).

[40] W. P. Tan, S. R. Souza, R. J. Charity, R. Donangelo, W. G. Lynch, and M. B. Tsang, Phys. Rev. C 68, 034609 (2003).

[41] J. P. Bondorf, A. S. Botvina, and I. N. Mishustin, Phys. Rev. C 58, R27 (1998).

[42] S. R. Souza, R. Donangelo, , W. G. Lynch, W. P. Tan, and M. B. Tsang, Phys. Rev. C 69, 031607(R) (2003).

[43] T. X. Liu, M. J. van Goethem, X. D. Liu, W. G. Lynch, R. Shomin, W. P. Tan, M. B. Tsang, G. Verde, A. Wagner, H. F. Xi, et al., Phys. Rev. C 69, 014603 (2004). 